\title{
Análisis espectral de materiales geológicos en la Cordillera Volcánica Central de Costa Rica y su relación con la detección remota de anomalías
}

\author{
Spectral analysis of geological materials in the Central \\ Volcanic Range of Costa Rica and its relationship to \\ the remote detection of anomalies
}

\author{
J.G. Rejas ${ }^{1,3}$, J. Martínez-Frías ${ }^{2}$, R. Martínez ${ }^{3}$, M. Marchamalo ${ }^{3}$, J. Bonatti ${ }^{4}$ \\ 1 Instituto Nacional de Técnica Aeroespacial, INTA. Email: rejasaj@inta.es \\ 2 Instituto de Geociencias (CSIC, UCM) \\ 3 Dpto. de Ingeniería y Morfología del Terreno, Universidad Politécnica de Madrid, UPM \\ 4 Universidad de Costa Rica, UCR
}

\section{RESUMEN}

El objetivo del presente trabajo es el estudio de bandas de absorción y reflexión de espectros de materiales geológicos presentes en distintas áreas de test de la Cordillera Volcánica Central (CVC) de Costa Rica, y su correlación con el cálculo automático de anomalías a partir de espectrometría de imagen de alta resolución, también llamada teledetección hiperespectral. En la detección de respuestas anómalas se asume un no conocimiento previo de los objetivos. Los píxeles se separan automáticamente en función de su información espectral diferenciada respecto de un fondo que se estima, bien de manera global para toda la escena, bien localmente por segmentación de la imagen o ventanas de búsqueda, para lo que resulta significativo la caracterización de patrones en base a las bandas diagnóstico de los materiales geológicos.

El estudio se ha llevado a cabo a partir de rangos del espectro electromagnético del visible e infrarrojo cercano, infrarrojo de onda corta y térmico de cubos de datos hiperespectrales procedentes de los sensores aeroportados HyMAP y MASTER. Se han diseñado experimentos sobre escenarios semiurbanos y naturales de la CVC de diferente complejidad, analizando el comportamiento del detector de anomalías estándar RX (Reed \& Xiaoli, 1990) y métodos distintos subespaciales y basados en proyección de imagen. Entre los métodos comparados con $\mathrm{RX}$ se propone uno nuevo denominado Detector de Anomalías de Fondo Térmico (DAFT), método que proyecta miembros puros espectrales sobre un subespacio térmico que define el fondo espectral, y que a su vez supone una variante de Projection Pursuit (Malpica et al., 2008). Se discuten los resultados obtenidos y se realiza una aproximación sobre las implicaciones en procesos geológicos, así como en análogos terrestres para exploración planetaria.

Palabras clave: análisis espectral; detección de anomalías; hiperespectral; reconocimiento automático de patrones; CVC; DAFT.

Recibido el 13 de febrero de 2014; Aceptado el 15 de mayo de 2014; Publicado el 3 de octubre de 2014

Citation / Cómo citar este artículo: Juan Gregorio Rejas et al. (2014). Análisis espectral de materiales geológicos en la Cordillera Volcánica Central de Costa Rica y su relación con la detección remota de anomalías. 70(2): e011. http://dx.doi.org/10.3989/ egeol.41711.313.

Copyright: ( 2014 CSIC. This is an open-access article distributed under the terms of the Creative Commons Attribution-Non Commercial (by-nc) Spain 3.0 License. 


\begin{abstract}
The aim of this work is the comparative study of methods for calculating spectral anomalies from imaging spectrometry in several test areas of the Central Volcanic Range (CVR) of Costa Rica. In the detection of anomalous responses it is assumed no prior knowledge of the targets, so that the pixels are automatically separated according to their spectral information significantly differentiated with respect to a background to be estimated, either globally for the full scene, either locally by image segmentation techniques.

We used spectral ranges of visible-near infrared (VNIR), shortwave infrared (SWIR) and thermal (TIR) data cubes hiperepectrales from HyMAP airborne sensors and MASTER. We have designed experiments on natural scenes of the CVC, and semi-urban of different complexity, analyzing the behavior of the standard RX (Reed \& Xiaoli, 1990) anomaly detector and different approaches based on imaging and dimensionality reduction. In this paper we propose a new technique for anomaly detection in hyperspectral data called DAFT (Detector de Anomalías de Fondo Térmico), as a Projection Pursuit (Malpica et al., 2008) variant, based on dimensionality reduction by projecting anomalies or targets with unknown spectral signature to the background, in a range thermal spectrum wavelengths. We discuss the results considering their implications on geological processes, as well as their use as terrestrial analogs for planetary exploration.
\end{abstract}

Keywords: spectral analysis; anomaly detection; hyperspectral; pattern recognition; CVR; DAFT.

\section{Introducción}

La espectrometría de imagen o teledetección hiperespectral explota el hecho de que todos los materiales reflejan, absorben y emiten energía electromagnética, con longitudes de onda específicas, en patrones distintivos relacionados con su composición molecular (Manolakis \& Shaw, 2002). Esta técnica proporciona información relacionada con las características superficiales de los materiales que pueden ser explotadas para permitir una detección automatizada de objetivos de interés, a partir de la construcción de un espectro de radiación o firma espectral esencialmente continua por cada píxel en la escena. Por lo tanto, la explotación de datos de espectrómetros de imagen hace posible la identificación a distancia de materiales del suelo de interés sobre la base de sus firmas espectrales.

Hay dos aplicaciones principales que dependen de la capacidad para separar los materiales basados en sus firmas espectrales: clasificación y detección de objetivos. El principal objeto de la clasificación es asignar automáticamente todos los píxeles de un cubo de datos hiperespectral a clases de cubiertas terrestres o temas, generando así cartografía temática. La detección de anomalías espectrales tiene por objeto sin embargo, extraer de manera automática píxeles de imagen que responden significativamente diferente respecto de su entorno. Distintos métodos se han desarrollado en las últimas décadas que han supuesto la mejora en el establecimiento de relaciones entre la dimensionalidad de los datos hiperespectrales y la optimización de los procesos de búsqueda (Duran \& Petrou, 2007; Schaum, 2005; Stein et al., 2002), así como la diferenciación subpíxel de la mezcla espectral y su implicación en las respuestas anómalas (Bar et al., 2010; Eismann et al., 2009; Manolakis \& Shaw, 2002).

En otro sentido, la espectrometría de imagen se ha mostrado eficaz para la caracterización de minerales en base a métodos estadísticos que utilizan las bandas reflectivas y absortivas diagnóstico de estos. Configuraciones hiperespectrales en rangos del infrarrojo de onda corta (SWIR), infrarrojo cercano y visible (VNIR) han permitido cartografiar materiales de alteración hidrotermal en distintos escenarios geológicos (Antón-Pacheco et al., 2001; Cipar et al., 2011; Crosta et al., 2003). Trabajos previos han demostrado la aplicación en mineralogía de rangos del espectro electromagnético en el infrarrojo térmico y fusión en la región de las microondas para detectar contaminación, materiales enterrados (Nasrabadi, 2008; Rejas et al., 2010) y otras aplicaciones de prospección del terreno.

Un aspecto relevante en nuestro estudio es establecer relaciones que permitan conectar las anomalías espectrales con lo que podemos denominar anomalías informacionales. Es decir, información relacionada con la respuesta anómala más allá de ser producto de una diferenciación significativa respecto de un fondo. Interesa por lo tanto conocer qué información geológica nos proporciona la respuesta anómala detectada y caracterizar en qué condiciones se produce. 
Se presenta en este sentido una aproximación multifuente, como estudio de la correlación entre anomalías espectrales y materiales de alteración hidrotermal en los complejos volcánicos de Turrialba e Irazú, situados en la Cordillera Volcánica Central (CVC) de Costa Rica. Así mismo se analiza la influencia de la resolución espectral en la determinación del fondo, aspecto crítico en el cálculo de anomalías. Se comparan los resultados con índices de vegetación y suelo en una zona con actividad geotérmica y vulcanológica creciente, por lo que, debido a la cercanía de núcleos urbanos, acrecientan la necesidad de un seguimiento en la evolución de su estado.

Finalmente, se avanza el estudio de bandas diagnóstico de espectros y su relación como valores atípicos en la detección de anomalías para un análisis espectral automatizado en Geología y Exploración Planetaria.

\section{Zona de estudio y preproceso de datos}

La zona de la CVC estudiada abarca una extensión de $98 \mathrm{~km}^{2}$, incluyendo los volcanes Irazú y Turrialba y sus entornos (Fig. 1), quedando encuadrada dentro del área delimitada por las esquinas de coordenadas geográficas (datum WGS84) latitud 9 9 59' $\mathrm{N}$ y longitud $83^{\circ} 51^{\prime} \mathrm{W}$ (esquina SW) y latitud $10^{\circ} 1^{\prime} \mathrm{N}$ y longitud $83^{\circ} 46^{\prime} \mathrm{O}$ (esquina NE).

Se han analizado datos de sensores aeroportados y satelitales en una estrategia multiescala. Se ha utilizado imágenes de reflectancia del sensor HyMAP (Cocks et al., 1998) y del sensor MASTER (Hook et al., 2001), adquiridas ambas el 7 de marzo de 2005 sobre la CVC.

La escena HyMAP (125 bandas entre $0.4589 \mu \mathrm{m} \mathrm{y}$ $2.491 \mu \mathrm{m})$ tiene una dimensión de $710 \times 2415$ píxeles, con una resolución espacial de $15 \mathrm{~m}$. La escena MASTER es de 1650×4466 píxeles, de resolución espacial de $9 \mathrm{~m}$ y codificación radiométrica 16 bit. Los 50 canales de imagen de MASTER se agrupan en un puerto de 25 canales en el VNIR-SWIR $(0.463 \mu \mathrm{m}$ a $2.427 \mu \mathrm{m})$, y 25 canales entre $3.075 \mu \mathrm{m}$ y $13 \mu \mathrm{m}$ del MIR-TIR.

Se han utilizado así mismo escenas ASTER comprendidas en un período entre 2002 y 2010, y una escena Hyperion del 5 de marzo de 2010 adquiridas a través de la plataforma Earth Explorer del United States Geological Survey -USGS (http://earthexplorer.usgs.gov/). Se ha aplicado un algoritmo MNF (Minimum Noise Fraction) que ha permitido reducir el ruido en las imágenes HyMAP y MASTER. Se han desechado los canales de los sensores que presentaban una relación señal/ ruido elevada, canales 62 a 65 y 125 en el caso de HyMAP, y canales 16 a 19, 25 a 41 y 50 para las imágenes MASTER.

Las imágenes aeroportadas han sido georreferenciadas directamente mediante el algoritmo GLT de ENVI (http://itt.envi.com), utilizando para ello la geometría calculada a partir de datos de posición y orientación medidos por sistemas inerciales basados en GPS (Global Positioning System) e IMU (Inertial Measurement Unit) en el momento de adquisición sobre el área de estudio. El error absoluto planimétrico obtenido ha sido de $3.2 \mathrm{y}$ 1.9 píxeles, respectivamente para las imágenes MASTER y HyMAP.

\section{Análisis exploratorios}

Se han aplicado técnicas exploratorias de imagen cuyo objetivo es contrastar parámetros biofísicos y obtener una primera aproximación del estado de la vegetación y los suelos en el entorno de los volcanes de la CVC estudiados.

Para ello, primeramente se ha estimado y minimizado el error de las imágenes, realizando un chequeo de las transformaciones radiométricas y geométricas a partir de datos medidos en campañas de supervisión. En agosto de 2010 y febrero de 2012 se llevaron a cabo reconocimientos sobre el terreno, que permitieron muestrear 29 y 20 puntos respectivamente, medidos en campo y en laboratorio con radiómetros USB400 y ASD FieldSpec Hi-Re. Los espectros se han utilizado para caracterizar materiales geológicos de alteración hidrotermal y para comprobar la reflectancia de las imágenes HyMAP y MASTER mediante modelos lineales de regresión empírica.

Se ha evaluado la influencia de la cubierta de vegetación, abundante en la zona de estudio, en el análisis espectral de imagen posterior, para estimar el impacto de la actividad de los complejos volcánicos en las laderas y zonas próximas. En este sentido, se han calculado primeramente índices de vegetación y de suelo para el conjunto de las 


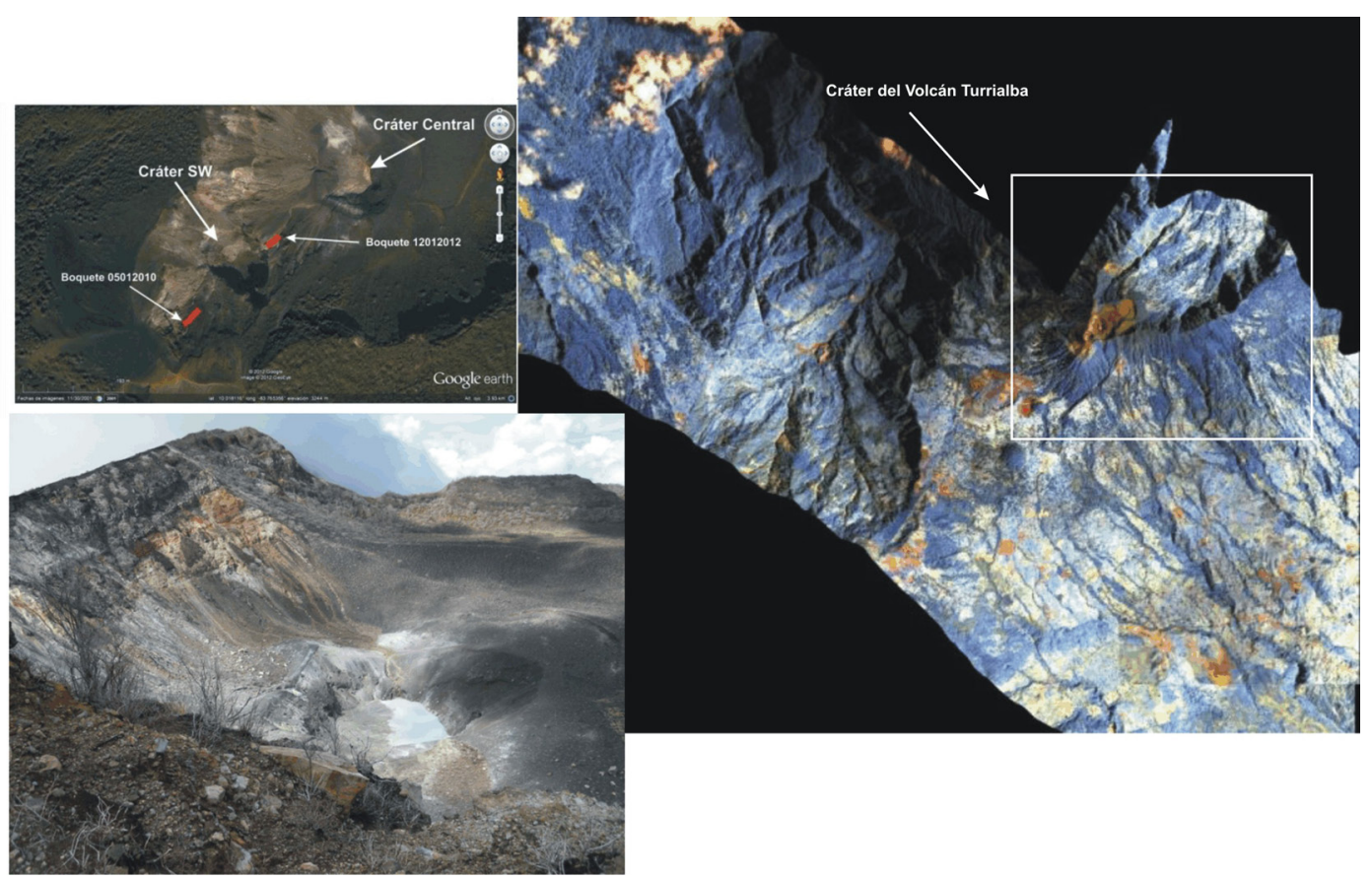

Fig. 1.-Cráter Central del volcán Turrialba (inferior izquierda) en 2010, complejo volcánico (izquierda superior) y mosaico de imágenes HyMAP RGB 12,25,36 de Turrialba en combinación de color de Rothery.
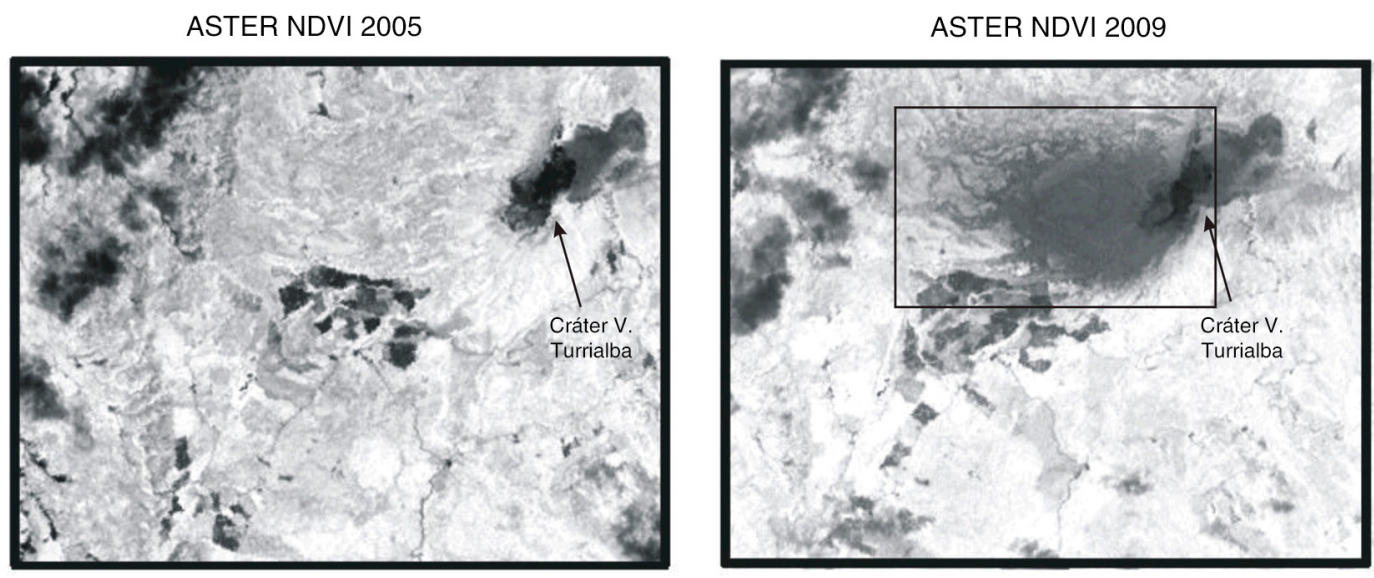

Fig. 2.-Efecto de la lluvia ácida (recuadro en imagen derecha) detectado mediante TCAR/OSAVI a partir de las imágenes ASTER de 2009, comparado con el mismo índice en la imagen ASTER de 2005 (izquierda).

imágenes. Se ha generado el NDVI o Normalized Difference Vegetation Index (Rouse et al., 1974) para evaluar el área foliar según la conocida expresión:

$$
N D V I=\frac{\rho_{\mu N I R}-\rho_{\mu R}}{\rho_{\mu N I R}-\rho_{\mu R}}
$$

donde $\rho_{\mu N I R}$ es la reflectancia de banda del infrarrojo próximo y $\rho_{\mu R}$ es la reflectancia de banda del rojo. Para estimar la reflectancia transformada por la absorción de la clorofila en relación la vegetación del suelo (Berni et al., 2010) se ha generado el ratio entre el índice TCARI (Transformed Chlorophyll Absorption Ratio Index) y OSAVI 
(Optimized Soil Adjusted Vegetation Index) particularizado para los sensores HyMAP y MASTER según la expresión:

$$
\begin{aligned}
& \text { TCARI / OSAVI }=3 \cdot\left[\left(\rho_{\mu 700}-\rho_{\mu 670}\right)-0.2 \cdot\left(\rho_{\mu 700}-\rho_{\mu 550}\right) \cdot\right] \\
&\left(\rho_{\mu 700}-\rho_{\mu 670}\right)(1+0.16) \cdot\left(\rho_{\mu 800}-\rho_{\mu 670}\right) / \\
&\left(\rho_{\mu 800}-\rho_{\mu 670}+0.16\right)
\end{aligned}
$$

donde $\rho_{\mu i}$ es reflectancia de la banda i.

Aprovechando la capacidad de separación entre las cubiertas del espectro térmico de MASTER y ASTER se han estudiado los patrones de emisividad de los materiales geológicos a partir del índice térmico THIN (Rejas et al., 2012):

$$
\operatorname{THIN}=\frac{\left[\operatorname{conv}\left(L_{\mu 10.16}\right)-\operatorname{conv}\left(L_{\mu 12.21}\right)\right]}{q \cdot\left[\operatorname{conv}\left(L_{\mu 10.16}\right)-\operatorname{conv}\left(L_{\mu 12.21}\right)\right]}
$$

donde conv es la imagen convolucionada y $L$ es la radiancia en el sensor por longitud de onda (en $10.16 \mu \mathrm{m}$ y $12.21 \mu \mathrm{m}$ ), siendo $q$ la razón entre las ganancias respectivas de los canales. La variable resultante se ha umbralizado para resaltar píxeles detectados como posibles patrones térmicos (Figura 3 (c)), que posibilitan la caracterización de los materiales geológicos para su posterior análisis en base a sus bandas diagnóstico de emisividad.

Los resultados obtenidos en estos análisis exploratorios de imagen han permitido, a partir de la serie temporal de escenas ASTER (Fig. 2), detectar y delimitar el efecto acusado de lluvia ácida sobre el vigor en la vegetación en una marcada dirección este-oeste debido a la actividad fumarólica.

\section{Alteración hidrotermal en la CVC}

Existen minerales claramente indicativos de la presencia de alteración hidrotermal que podemos detectar remotamente a partir de su respuesta espectral en bandas diagnóstico de absorción y reflexión (Antón-Pacheco et al., 2001; Bataller et al., 2010). Estas características espectrales que los hacen únicos, las podemos extraer de imágenes hiperespectrales convenientemente calibradas. Minerales arcillosos, tales como caolinita, illita y alunita, presentan una alta reflectancia entre las longitudes de onda de $1,55 \mu \mathrm{m}$ y $1,75 \mu \mathrm{m}$, y una alta absorción entre de $2,08 \mu \mathrm{m}$ y $2,35 \mu \mathrm{m}$. Otra característica adicional para identificar estos minerales es que las rocas que no han sufrido el proceso hidrotérmico, por lo general presentan valores normales en las longitudes de onda antes mencionadas. Los minerales con alto contenido en Fe presentan un contraste muy elevado entre las longitudes de
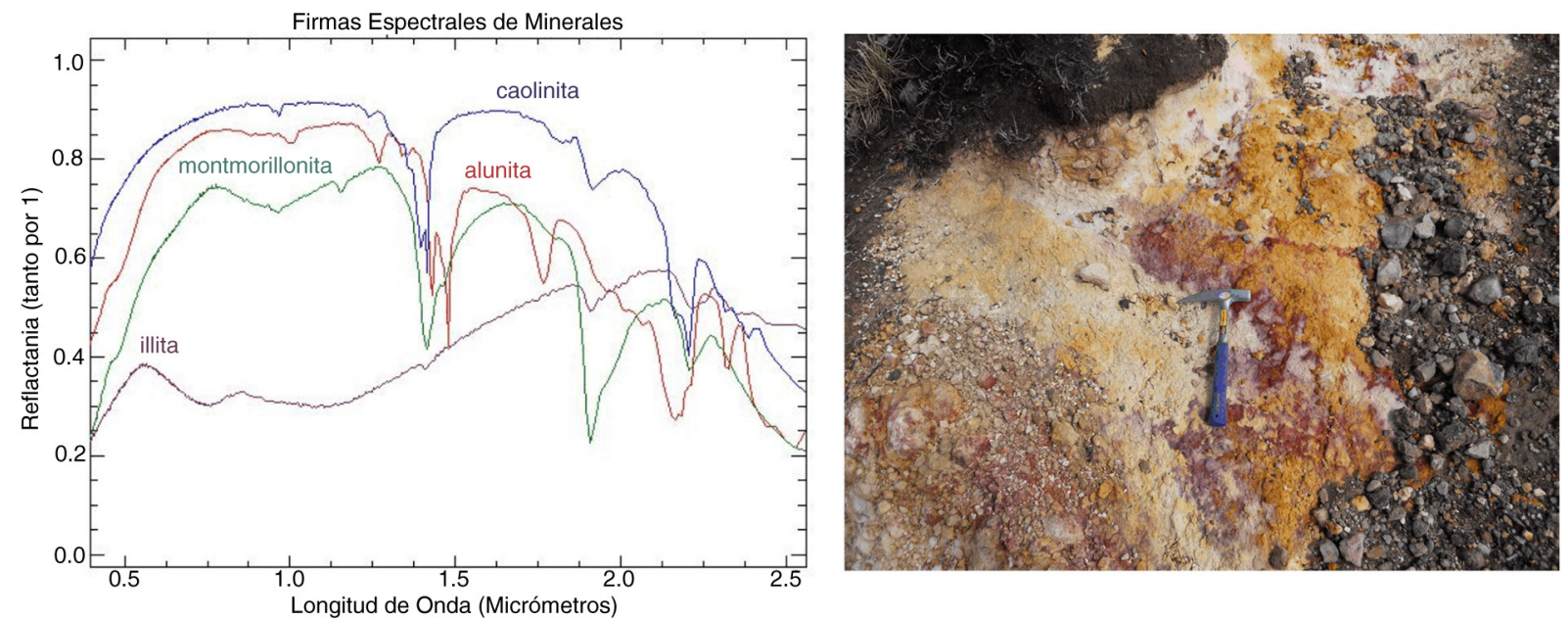

Fig. 3.-Espectros de reflectancia extraídos de las imágenes calibradas de HyMAP (izquierda) y alteración hidrotermal en el cráter del volcán Turrialba en 2010 (derecha). 
onda $0,63 \mu \mathrm{m}$ y $0,69 \mu \mathrm{m}$ y longitudes de onda de $0,45 \mu \mathrm{m}$ a las $0,52 \mu \mathrm{m}$.

En base a la medida y análisis espectral de las firmas obtenidas de muestras tomadas en la CVC, hemos seleccionado cuatro variables de imagen para realizar un análisis de componentes principales (ACP), o en inglés Principal Components Analisys (PCA), diferenciados por grupos de bandas, dos altamente reflectantes y dos altamente absorbentes para cada mineral (Crosta \& Moore, 1989; Crosta et al., 2003). Esta técnica favorece la identificación de nuevas variables imagen o componentes principales (PCs) que contienen información espectral concreta para materiales específicos, al igual que la contribución de cada una de las bandas originales relacionadas a la respuesta espectral teórica de los materiales buscados con base en el signo y en la magnitud de los vectores característicos.

En el caso de las imágenes HyMAP se han seleccionado las siguientes bandas en los sucesivos PCA: para illita, canales 6, 25, 105 y 108; para alunita canales $6,25,105$ y 116; para caolinita canales 6,81 , 108 y 116; para caolinita + esmecita canales 6, 81, 108, 119. Para el grupo de minerales de Fe, como la goethita, hematita y jarosita, se han utilizado los canales HyMAP 4, 32, 62 y 103 y para la montmorillonita los canales 4, 25, 81 y 94 .

En el caso de las imágenes MASTER se han realizado análisis de componentes principales diferenciados para cada mineral a partir de las siguientes longitudes de onda: canales 4, 8, 21 y 24 para alunita; canales 4, 8, 21 y 22 para illita; canales 4, 13, 22 y 24 para caolinita.

Los resultados obtenidos para HyMAP y MASTER (Fig. 4 izqda. y centro, respectivamente) se han clasificado mediante el algoritmo K-Means, calculando matrices de confusión a partir del muestreo de campo adoptado como valor real. Para HyMAP se ha obtenido un solape de precisión al $82.56 \%$ y coeficiente Kappa de 0.75 . Para MASTER se ha obtenido un solape de precisión al $75.11 \%$ y coeficiente Kappa de 0.69 . Los resultados están muy influenciados por la cubierta vegetal, que actúa como aporte erróneo de reflectancia en el cálculo de las nuevas variables o CPs. Sin embargo, se observa la escasa presencia de materiales arcillosos de alteración fílica, lo que contrasta con la abundancia de los componentes de óxido de hierro.

\section{Detección de anomalías espectrales en la CVC}

Se ha probado en el conjunto de las imágenes el algoritmo RX (Reed \& Xaoli, 1996) admitido ampliamente como un estándar en la detección de anomalías espectrales en su modalidad global. Se han contrastado las anomalías obtenidas por RX con las calculadas mediante DAFT como variante de Projection Pursuit (Malpica et al., 2008), mediante un método SSRX basado en subespacios hiperespectrales (Borghys et al. 2012) y mediante ortoproyección subespacial - OSPRX (Chang, 2005).

El algoritmo RX básico formula dos hipótesis: la primera modeliza el fondo de la imagen como una distribución Gaussiana de media cero y una matriz de covarianza desconocida que es estimada globalmente o localmente a partir de los datos. La segunda hipótesis modeliza la anomalía como una combinación lineal de una signatura objetivo y un ruido de fondo, maximizando la distancia de Mahalanobis. En SSRX, se aplica el RX global en un número limitado de bandas de PCA. Los primeros componentes (PCs) son desechados o utilizados como fondo espectral en SSRX. En el método OSPRX, los primeros componentes de PCA definen el fondo subespacio y los datos se proyectan en el subespacio ortogonal antes de aplicar el detector de RX.

El método DAFT se implementa en cuatro fases: (1) definición de subespacios espectrales, (2) extracción de miembros puros en el subespacio VNIR, (3) proyección sobre el fondo térmico y reducción de la dimensionalidad, y (4) búsqueda de la mejor proyección maximizando el índice térmico THIN. La secuencia de fases en DAFT comienza por lo tanto con la generación de subespacios desde el cubo de datos hiperespectral, formando un subespacio espectral del visible hasta $2.5 \mu \mathrm{m}$ de longitud de onda, y otro desde los $3 \mu \mathrm{m}$ en adelante hasta el espectro térmico. En este método las primeras variables de PCA particularizado para las longitudes de onda entre 3 y $14 \mu \mathrm{m}$, definen el subespacio del fondo espectral. Los datos correspondientes al puerto VNIR se proyectan entonces en el subespacio térmico antes de maximizar el "interés" de THIN (3) adoptando el estándar RX, en su variante global o local. 

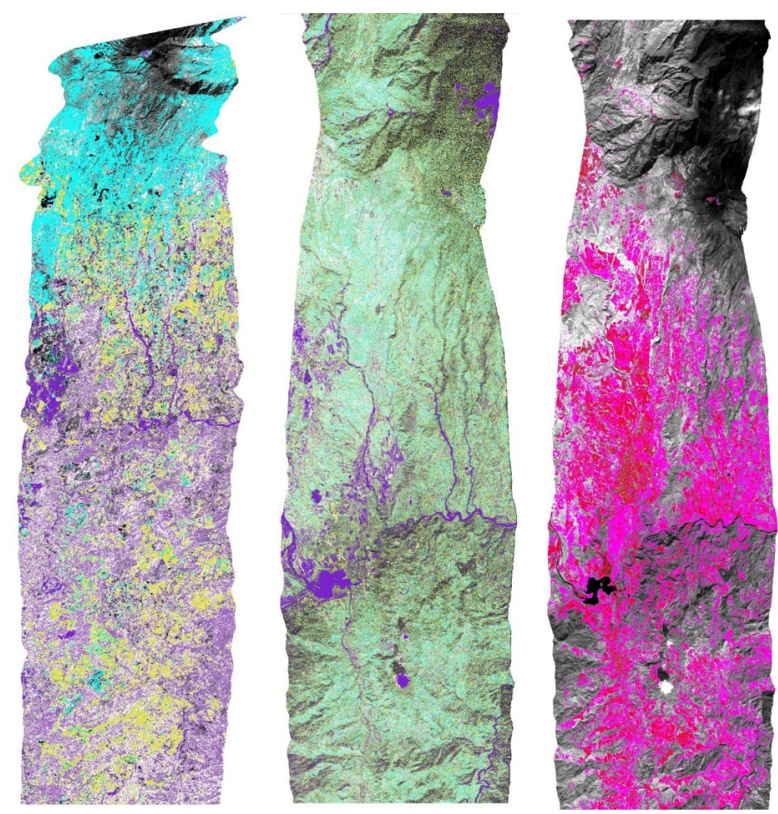

Fig. 4.-Abundancia de minerales de alteración hidrotermal (Alu-cyan, Illi-amarillo, Caol-morado, Caol+Esme-morado 2, Mont-marrón) a partir de HyMAP (izquierda) y MASTER (centro) para la escena de imagen del volcán y ciudad de Turrialba. Índice térmico normalizado THIN (rojo-violeta) entre longitudes de onda de $10.16 \mathrm{~mm}$ y $12.21 \mathrm{~mm}$ superpuesto sobre canal 6 en nivel de gris de MASTER (derecha).

Para todos los métodos se ha separado el cálculo por rangos de canales reflectivos y emisivos en el caso de las imágenes MASTER (Fig. 4 dcha.) y ASTER, focalizando en los canales térmicos fusionados con fotografía aérea a $2.5 \mathrm{~m}$ de resolución, para mejorar la separabilidad de clases manteniendo la información espectral (Rejas et al., 2007), observándose que la nueva variable 6 proyectada registra menor concentración de humedad.

Aunque los resultados son muy similares en todos los métodos para los valores más altos del umbral, el método de DAFT es más preciso en la detección de anomalías para los valores más bajos del umbral correspondientes a materiales altamente separados por su emisividad. Por ejemplo, la Figura 5 (d) de la columna derecha muestra cómo DAFT separa el agua del cráter del volcán Irazú de las anomalías de las paredes del cráter, mientras que en SSRX y OSPRX se confunden. En la columna izquierda, se observa como DAFT separa más claramente las anomalías débiles (en rojo) asociadas a cubiertas de vegetación respecto de los minerales, mientras que en SSRX sobre todo y en OSPRX la vegetación se detecta como anomalía débil confundiéndolo con el fondo espectral. En realidad, todo lo que tiene una firma espectral que es diferente de la mayoría de las del promedio del fondo, se detecta como anomalía por todos los métodos, pero en DAFT se optimiza la separación a partir de la emisividad de los materiales. Cuanto más diferente es la firma, más alto es el valor de la detección.

\section{Discusión de resultados}

Se ha estudiado la relación entre las anomalías espectrales y las bandas diagnóstico de las alteraciones hidrotermales obtenidas en los anteriores apartados. El estudio ha consistido por un lado en la extracción de información mineralógica y presencia de determinados compuestos mediante análisis separados de componentes principales en la llamada Técnica de Crosta. Varios de estos minerales se han detectado remotamente en los test de Irazú y Turrialba a partir de sus respuestas espectrales de absortancia y reflectancia. Pretendemos responder a la pregunta de si existe correlación entre la detección de anomalías por diferentes métodos y la consideración o no en el cálculo computacional de la radiancia en las longitudes de onda donde determinados minerales se caracterizan sin equívoco (Fig. 6). Para ello, se ha identificado primeramente esas bandas diagnóstico, para seguidamente registrar el valor de la anomalía obtenida por cada método.

Para ello se ha ajustado linealmente un espacio muestral de 35 pares de puntos, anomalías espectrales en el eje Y y minerales alterados en el eje X. Todos las regresiones se han calculado a un nivel de confianza del $95 \%$, eliminando en cada ajuste los valores muestrales que presentaban residuos inusuales, que se correspondían principalmente con nubes y sombras. Los resultados obtenidos para OSPRX global y DAFT global se resumen en las Tablas 1 y 2 , respectivamente.

De los modelos lineales generados, el que obtiene un más alto valor de $\mathrm{R}$ cuadrado de $73.12 \%$ se corresponde con el de DAFT- Illita para las imágenes de MASTER.

El valor P obtenido inferior a 0.05 en todos los casos, salvo para OSPRX-caolinita y OSPRXmontmorollonita a partir de HyMAP, indica que hay 
(a)
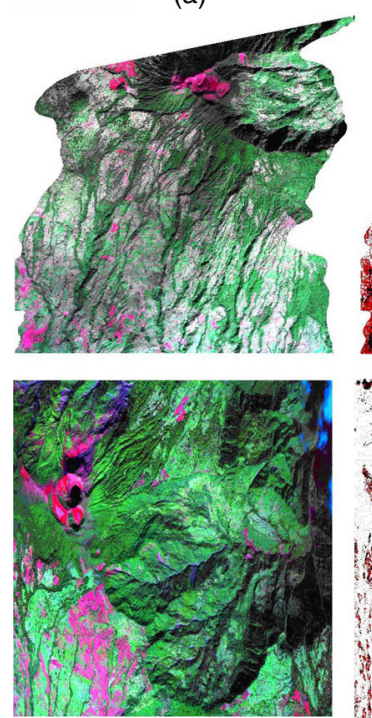

(b)

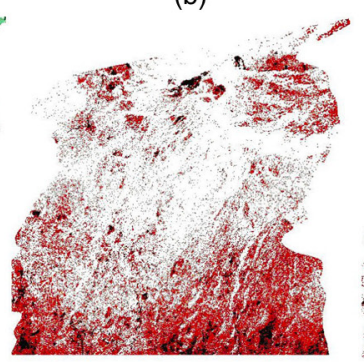

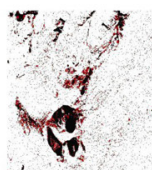

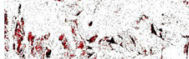
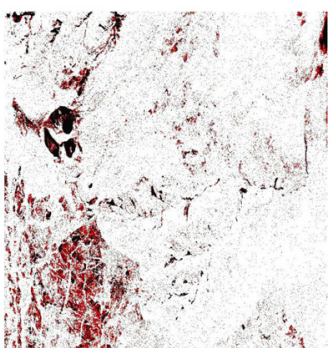
Wit.
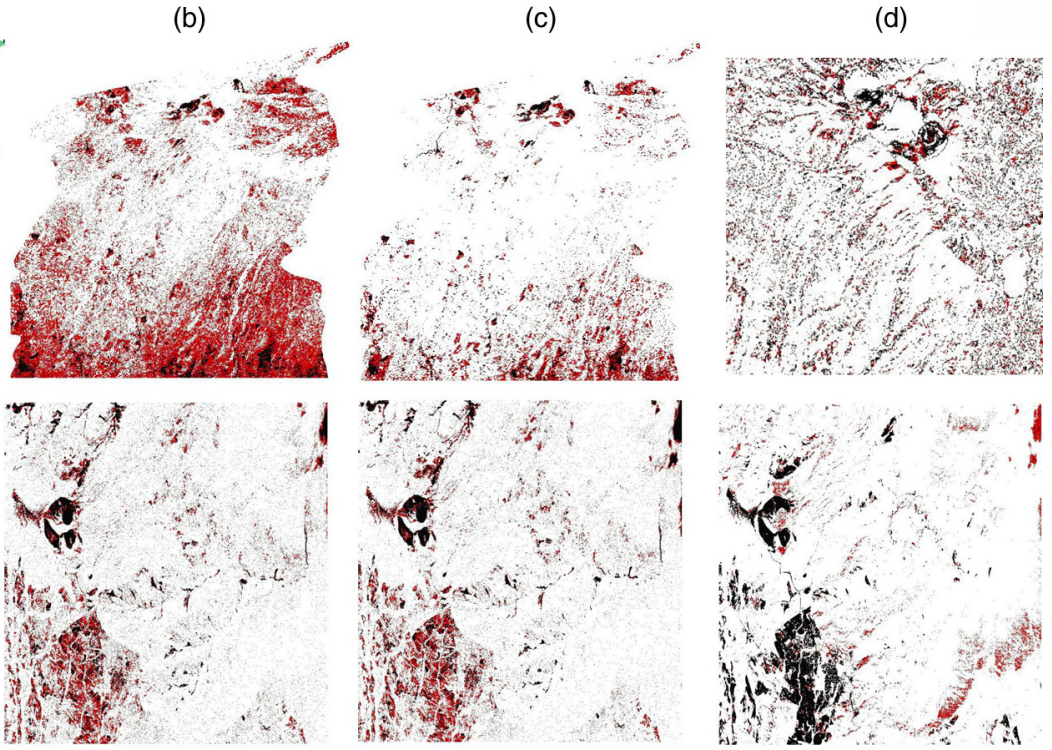

Fig. 5.-En fila superior: imagen HyMAP en combinación de color RGB 118,49,67 del Volcán Turrialba (a), anomalías espectrales fuertes (en negro) obtenidas mediante SSRX global (b), OSPRX global (c) y DAFT obtenido de las imágenes MASTER de Turrialba (d). En fila inferior: imagen MASTER en combinación de color RGB 43,11,22 del Volcán Irazú (a) y anomalías espectrales fuertes (en negro) obtenidas mediante SSRX global (b), OSPRX global (c) y DAFT (d).
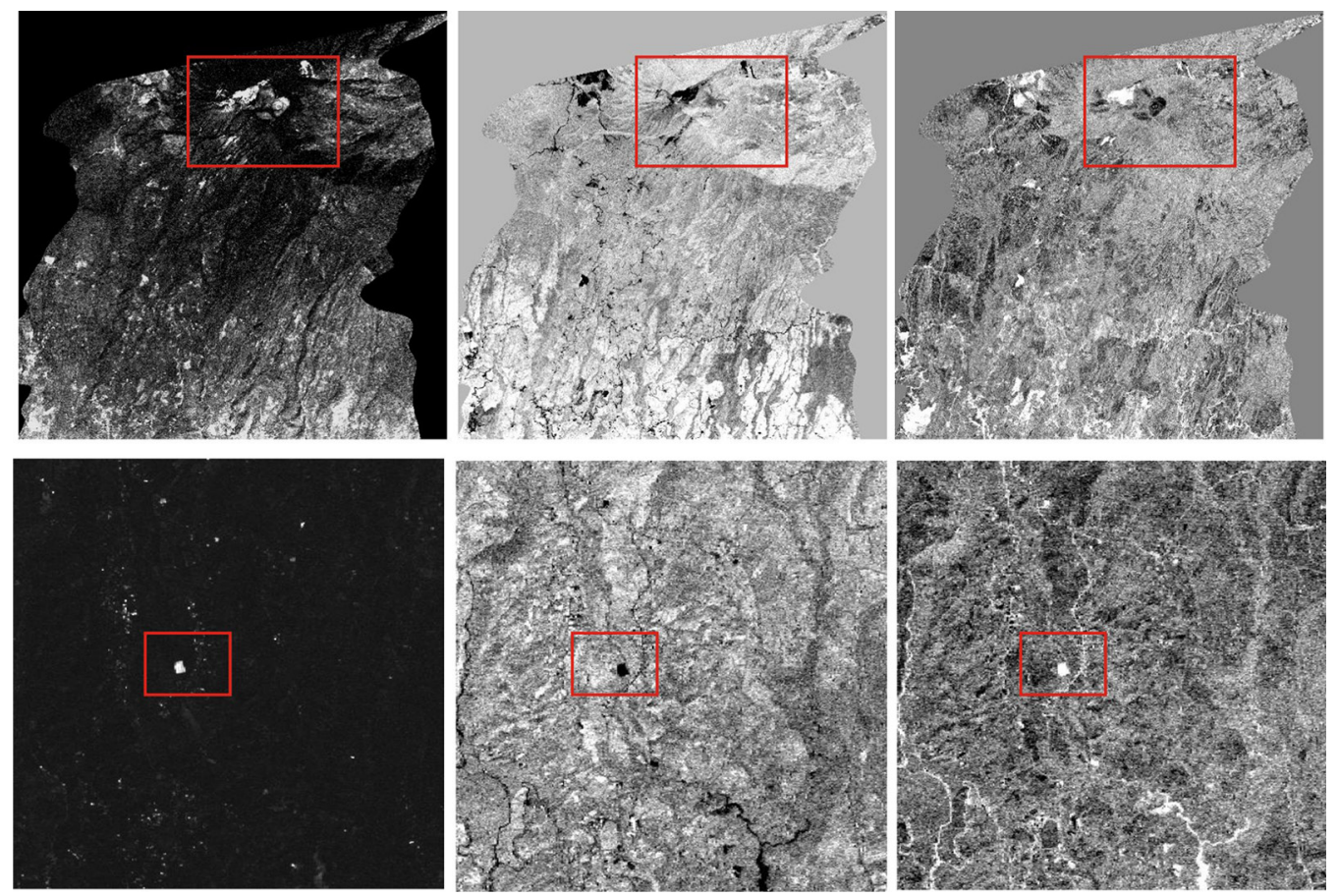

Fig. 6.-Detalle en el Volcán Turrialba de anomalías espectrales SSRX (superior-izquierda), Alunita (sup.-centro) e Illita (sup.-derecha) a partir de HyMAP 2005. Detalle de anomalías espectrales OSPRX (inferior-izquierda), Alunita (inf.-centro) e Illita (inf.-derecha) a partir de MASTER 2005 en las laderas del Volcán Turrialba.

una relación estadísticamente significativa y no hay ninguna indicación de autocorrelación serial en los residuos en el nivel de confianza del 95.0\%.
El estadístico R-cuadrado indica que los modelos ajustados explican en diferentes porcentajes la variabilidad entre alteración hidrotermal y anomalías 

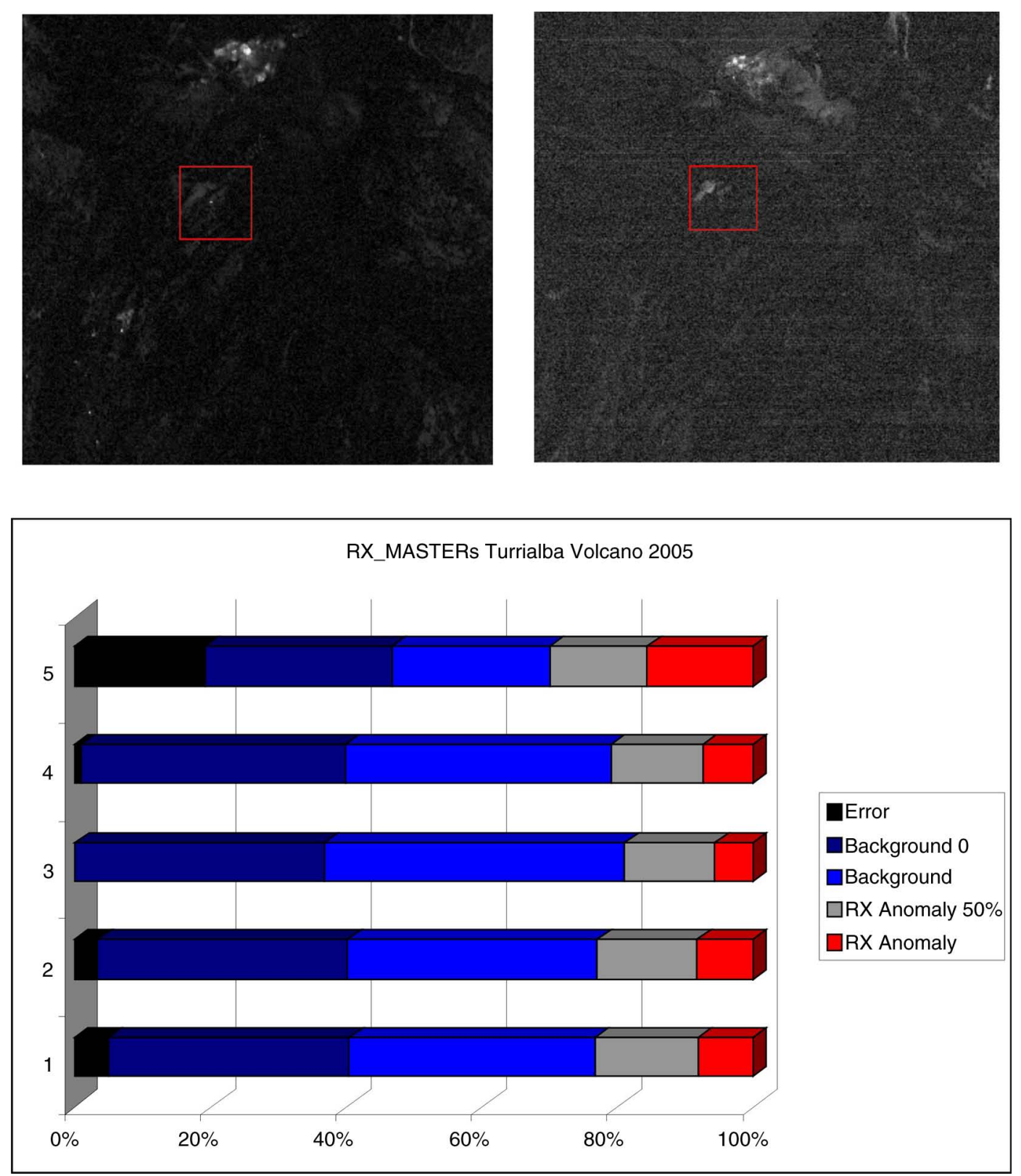

Fig. 7.-Anomalías RX de canales VNIR (sup.-izquierda) y TIR a partir de MASTER (sup.-derecha). Comparación (inferior) de métodos de detección de anomalías RX para MASTER: (1) Todos los canales, (2) sólo VNIR, (3) sólo bandas VNIR diagnóstico, (4) sin bandas VNIR diagnóstico y (5) sólo TIR.

Tabla 1.-Relación de anomalías OSPRX y alteración hidrotermal a partir de HyMAP

\begin{tabular}{lccc}
\hline Modelos de regresión HyMAP & P-valor & Correlación & \multicolumn{1}{c}{$R^{2}$} \\
\hline OSPRX $=1101,61+9,86744^{*}$ Alunita & 0,0001 & 0,850365 & $72,31 \%$ \\
OSPRX $=-1528,05-1,92404^{*}$ Illita & 0,0379 & $-0,554625$ & $30,76 \%$ \\
OSPRX $=-611,046+1,03826^{*}$ Caolinita & 0,0515 & 0,511091 & $26,12 \%$ \\
OSPRX $=739,318-7,59725^{*}$ Caol+Esmecita & 0,0182 & $-0,599314$ & $35,92 \%$ \\
OSPRX $=834,65+3,50578^{*}$ minerales Fe & 0,0153 & 0,611924 & $37,45 \%$ \\
OSPRX $=-796,88-1,47957^{*}$ Montmorollonita & 0,0851 & $-0,45922$ & $21,09 \%$ \\
\hline
\end{tabular}


Tabla 2.-Relación entre anomalías DAFT y alteración hidrotermal a partir de MASTER

\begin{tabular}{lllc}
\hline Modelos de regresión MASTER & $P$-valor & Correlación & $R^{2}$ \\
\hline DAFT $=79,4678-0,579643^{*}$ Alunita & 0,0004 & $-0,811637$ & $65,88 \%$ \\
DAFT $=69,1488-0,50982^{*}$ Illita & 0,0001 & $-0,855083$ & $73,12 \%$ \\
DAFT $=126,893-0,905153^{*}$ Caolinita & 0,0178 & $-0,620723$ & $38,53 \%$ \\
\hline
\end{tabular}

espectrales. Los coeficientes de correlación máximos $(-0.831632$ y 0.850365$)$ indican una relación moderadamente fuerte entre las correspondientes variables.

Existe correlación, positiva o negativa dependiendo del caso, entre las alteraciones hidrotermales detectadas y las anomalías espectrales calculadas a partir de los canales reflectivos de HyMAP. En el caso de las anomalías térmicas, no es tan consistente esta relación. Sin embargo, ésta si se evidencia con parámetros biofísicos calculados y con efectos asociadas a la actividad de los volcanes. Un caso particular es el del propio cráter, en el que esta actividad se detecta como anomalía térmica, pero pasa casi desapercibida como anomalía espectral en el rango reflectivo.

La alteración hidrotermal detectada a partir de HyMAP en CVC y entorno, está vinculada a áreas de cultivos o suelo desnudo y a superficies artificiales (cubiertas de edificaciones principalmente). Esto no implica que en los alrededores de estas zonas no existan minerales de alteración hidrotermal. Se observa que la detección está fuertemente influenciada por el aporte de la amplia, densa y homogénea cobertura natural de vegetación en la información espectral registrada por los sensores espaciales.

Se ha analizado la influencia de las bandas de absorción y reflexión en la detección de anomalías espectrales por los diferentes métodos mencionados (Fig. 7). La diferencia entre las anomalías espectrales con y sin bandas de diagnóstico en el VNIR para el caso HyMAP es el $0,75 \%$, y para el caso de MASTER es el $0,84 \%$, mientras que la diferencia para la detección del fondo espectral es de $-2,52 \%$ y $-2,32 \%$, respectivamente.

Se ha comprobado la influencia de las bandas diagnóstico en la detección de anomalías. Si bien, los minerales por su composición cristalina y el traspaso de cargas entre iones (Van der Meer \& Jong, 2001) mantienen una interacción muy característica y típica con la energía electromagnética en longitudes de onda principalmente del SWIR, hacen de ellos sujetos muy favorables donde se produce esta influencia. Esto responde de nuevo al fundamento estadístico y a la localización de valores atípicos en determinados grupos de bandas espectrales.

\section{Conclusiones}

Se ha realizado un estudio comparativo de distintos métodos de detección de anomalías espectrales a partir de imágenes HyMAP y MASTER en áreas de test de la Cordillera Volcánica Central (CVC) de Costa Rica correspondientes a los volcanes Irazú y Turrialba y sus entornos.

Se ha comprobado que la mayor resolución espectral de las imágenes mejora la precisión en la detección de bandas diagnóstico de los minerales de alteración hidrotermal analizados. Una mayor dimensionalidad de los datos implica no obstante un mayor número de muestras para caracterizar clases de materiales.

Mayores concentraciones de minerales de alteración hidrotermal, en escenarios en los que las fuentes de error (principalmente la vegetación) se minimizan, están correlacionadas con las anomalías calculadas en el rango espectral reflectivo. No se ha establecido una relación clara entre anomalías exclusivamente térmicas y minerales analizados.

La mezcla espectral, asociada directamente con la resolución espacial de los sensores aeroportados y satelitales utilizados, repercute significativamente en la caracterización del fondo, y consiguientemente en las anomalías espectrales calculadas.

Los resultados obtenidos por los distintos métodos de detección de anomalías espectrales desarrollados se ven afectados en su posible correlación con lo que hemos denominado anomalías informacionales, es decir, con materiales, superficies y coberturas concretos, por la selección, automática o basada en experto, de bandas de absorción y reflexión en la fase de reducción de la dimensionalidad. Se ha 
comprobado, en línea con lo investigado en (Hsuan \& Chang, 2003) o (Rejas et al., 2012) como los resultados y la probabilidad de detección de verdaderos positivos y el rendimiento mejoran al considerar en el cálculo la respuesta en longitudes de onda diagnóstico y de manera significativa para determinados materiales geológicos. Ello permite considerar como adecuadas determinadas áreas de la CVC para análogos terrestres en exploración Planetaria mediante teledetección hiperespectral.

\section{AGRADECIMIENTOS}

Los autores agradecen al JPL, Jet Propulsion Laboratory de NASA por facilitar parte de los datos empleados en el estudio así como al USGS, United States Geological Survey por facilitar los datos Hyperion y las librerías espectrales de minerales utilizadas.

$\mathrm{Al}$ Centro de Astrobiología (CAB) los autores agradecen el análisis espectral de firmas en laboratorio así como el análisis geoquímico de las muestras obtenidas en las campañas de 2010 y 2012 en las áreas de experimentación de la Cordillera Volcánica Central de Costa Rica.

\section{Referencias}

Antón-Pacheco, C.; Rowan, L.C.; Mars, J.C. \& Gumiel, J.C. (2001). Characterization of mine materials and hydrothermally altered rocks in the río Tinto minning districy (southwest Spain) using HyMAP data. Revista de Teledetección, 16: 65-68.

Bar, D.O.; Wolowelsky, K.; Swirski, Y.; Figov, Z.; Michaeli, A.; Vaynzof, Y.; Abramovitz, Y.; BenDov, A.; Yaron, O.; Weizman, L. \& Adar, R. (2010). Target detection and verification via airborne hyperspectral and high-resolution imagery processing and fusion. IEEE Sensors Journal, 10(3): 707-711. http:// dx.doi.org/10.1109/JSEN.2009.2038664.

Bataller, F.J.; Rejas, J.G.; Bonatti, J.; Marchamalo, M. \& Martínez, R. (2010). Detection of hydrothermal alteration using a principal component analysis applied to hyperespectral HyMAP data on the Turrialba volcano, Costa Rica. Geomatica Week International Congress, Barcelona (Spain).

Berni, J.A.; Zarco-Tejada, P.; Suárez, L. \& Fereres, E. (2010). Thermal and narrowband multispectral remote sensing for vegetation monitoring from an Unmanned Aerial Vehicle. IEEE Transactions On Geoscience And Remote Sensing, 47(3): 722-738.

Borghys, D.; Achard, V.; Kasen, I. \& Perneel, C. (2012). Comparative evaluation of hyperspectral anomaly detection methods in scenes with diverse complexity. OPTRO2012 Proceedings, Symposium on Optronics in Defence and Security, Paris (France).
Chang C.I. (2005). Orthogonal subspace projection (OSP) revisited: a comprehensive study and analysis. IEEE Transactions on Geoscience and Remote Sensing, 43(3): 502-518. http://dx.doi.org/10.1109/ TGRS.2004.839543.

Cipar, J.; Anderson, G. \& Cooley, T. (2011). Active volcano monitoring using a space-based short-wave infrared imager. WHISPERS 2011 Proceedings, Lisbon (Portugal).

Cocks T.; Jenssen, R.; Stewart, A.; Wilson, I. \& T. Shields, T. (1998). The HyMAP Airborne Hyperspectral Sensor: The System, Calibration and Performance. 1st EARSeL Workshop on Imaging Spectroscopy, Zurich.

Crosta, A.P.F.; de Souza, C.R.; Azevedo, F. \& Brodie, C. (2003). Targeting key alteration minerals in epithermal deposit in Patagonia, Argentina, using ASTER imagery and principal component analysis. International Journal of Remote Sensing, 10: 4233-4240. http://dx.doi.org/10.1080/0143116031000152291.

Crosta, A.P. \& Moore, J. McM. (1989). Enhancement of Landsat Thematic Mapper imagery for residual soil mapping in SW Minas Gerais State Brazil: a prospecting case history in greenstone belt terrain. Proceedings of the 9th Thematic Conference on Remote Sensing for Exploration Geology, Calgary, 1173-1187.

Duran, O. \& Petrou, M. (2007). A time-efficient method for anomaly detection in hyperspectral images. IEEE Transactions on Geoscience and Remote Sensing, 45(12): 3894-3904. http://dx.doi.org/10.1109/ TGRS.2007.909205.

Eismann, M.T.; Stocker, A.D. \& Nasrabadi, N.M. (2009). Automated Hyperspectral Cueing for Civilian Search and Rescue. Proceedings of the IEEE, 97(6): 1031-1055. http://dx.doi.org/10.1109/JPROC.2009. 2013561.

Hook, S.J.; Myers, J.J.; Thome, K.J.; Fitzgerald, M. \& Kahle, A.B. (2001). The MODIS,ASTER airborne simulator (MASTER) - a new instrument for earth science studies. Remote Sensing of Environment, 76(1): 93-102. http://dx.doi.org/10.1016/ S0034-4257(00)00195-4.

Hsuan, R. \& Chang, C. (2003). Automatic spectral target recognition in hyperspectral imagery. Aerospace and Electronic Systems IEEE Transactions, Vol. 39(4): 1232-1249.

Manolakis, D. \& Shaw, G. (2002). Detection Algorithms for Hyperspectral Imaging Aplications. IEEE Signal Processing Magazine, 19(1): 29-43. http://dx.doi. org/10.1109/79.974724.

Malpica, J.A.; Rejas, J.G. \& Alonso, M.C. (2008). A projection pursuit algorithm for anomaly detection in hyperspectral imagery. Pattern Recognition 41(11): 3313-3327.

Nasrabadi, N.M. (2008). Multisensor Joint Fusion and Detection of Mines Using SAR and Hyperspectral. 
Sensors 2008 IEEE, 1056-1059. http://dx.doi. org/10.1109/ICSENS.2008.4716625.

Reed, I.S. \& Xiaoli, Y. (1990). Adaptative multiple-band CFAR detection of an optical pattern with unknown spectral distribution. IEEE Transactions on Acoustics, Speech and Signal Processing, 38(10): 1760-1770. http://dx.doi.org/10.1109/29.60107.

Rejas, J.G.; Martínez R. \& Malpica J.A. (2007). Hyperspectral remote sensing application for semi-urban areas monitoring. Urban Remote Sensing Joint Event 2007, Paris (France). http://dx.doi.org/ 10.1109/URS.2007.371806.

Rejas, J.G.; Burillo, F.; López, R.; Cano, M.A.; Sáiz, M.E.; \& Farjas, M. (2010). Integrating SAR data and hyperspectral analysis for the archaeological survey of the Segeda city, Spain. 3th International Conference on Remote Sensing in Archaeology, Tiruchirapalli (India). BAR International Series, 2118: 33-36.

Rejas, J.G.; Martínez-Frías, J.; Bonatti, J.; Martínez, R. \& Marchamalo, M. (2012). Anomaly detection and comparative analisys of hydrothermal alteration materials trough hyperspectral multisensor data in the Turrialba volcano. XXII ISPRS International Conference, Melbourne, Australia.

Rondeaux, G.; Steven, M. \& Baret, F. (1996). Optimization of soil-adjusted vegetation indices. Remote Sening of Environment, 55(2): 95-107. http://dx.doi. org/10.1016/0034-4257(95)00186-7.

Rouse, J.W.; Haas, R.H.; Schell, J.A.; Deering, D.W. \& Harlan, J.C. (1974). Monitoring the vernal advancements and retrogradation of natural vegetation. NASA/GSFC, Greenbelt, $371 \mathrm{pp}$.

Schaum, A. (2005). Hyperspectral detection algorithms: Operational, next generation, on the horizon. Proceedings of the 34th Applied Imagery and Pattern Recognition Workshop (AIPR05).

Stein, D.W.J.; Beaven, S.G.; Hoff, L.E.; Winter, E.M.; Schaum, A.P. \& Stocker, A.D. (2002). Anomaly Detection from Hyperspectral Imagery. IEEE Signal Processing Magazine, 19(1): 58-69. http://dx.doi. org/10.1109/79.974730.

Van der Meer, F.D. \& Jong, S.M. (Eds.) (2001). Imaging Spectrometry. Basic Principles and Prospective Applications. Springer, Netherlands. Remote Sensing and Digital Image Processing, 4: 403 pp. 